\section{Evaluation of choroidal thickness using spectral- domain optical coherence tomography in migraine patients during acute migraine attacks: a comparative study}

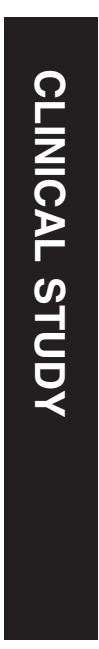

\begin{abstract}
Purpose The purpose of this study was to assess choroidal thickness in migraine patients during acute migraine attacks and compare them with healthy controls, using spectral domain optical coherence tomography (OCT).

Patients and methods In this prospective case-control study, choroidal thicknesses of 46 migraine patients during acute migraine attacks and 46 age- and sex-matched healthy subjects were measured using a high-speed, high-resolution frequency domain-OCT device. All patients underwent a complete ophthalmic examination before the measurements. OCT measurements were taken at the same time of day (0900 hours), in order to minimize the effects of diurnal variation.

Results There was a statistically significant difference in mean choroidal thickness between the migraine patients during acute migraine attacks $(356.3 \pm 21.46 \mu \mathrm{m})$ and the controls $(302.3 \pm \mathbf{1 8 . 3 4} \mu \mathrm{m} ; P=\mathbf{0 . 0 0 0})$. There were significant differences at all measurement points $(P<\mathbf{0 . 0 0 1}$ for all). Conclusion The increased choroidal thickness of the migraine patients during acute migraine attacks might be related to the vascular pathology of the disease. Further studies are needed to evaluate the etiopathologic relationship between choroidal thickness and acute migraine attack.
\end{abstract}

A Karalezli ${ }^{1}$, C Simsek ${ }^{2}$, G Celik ${ }^{3}$ and FC Eroglu ${ }^{1}$

Eye (2014) 28, 1477-1481; doi:10.1038/eye.2014.218;

published online 26 September 2014

\section{Introduction}

Migraine is a common neurologic syndrome characterized by recurrent, moderate, or severely intense headaches that can be uni- or bilateral. Headache duration changes between hours and days, and is usually accompanied by nausea, photophobia, and phonophobia that worsen by routine physical exertion. Two competing theories dominate the migraine pathogenesis discussion. First one, the vasogenic theory, views migraine as a form of vascular dysregulation and assumes that the aura is due to a transient vasoconstrictioninduced hypoxemia. ${ }^{1}$ Second, the alternative neurogenic theories, views migraine as a cranial disorder in which vascular changes are the result of neuronal dysfunction. ${ }^{2}$

According to the vasogenic theory, migraine headache is caused by a rebound vasodilatation that results in a mechanical depolarization of primary nociceptive neurons within the walls of engorged intra- and extracerebral vessels.

Following the early vasoconstrictive stage, meningeal blood vessels dilate, activating the trigeminal sensory nerves that surround them, causing pain. This activation of trigeminal nerves also causes the release of vasoactive neuropeptides, further contributing to dilation and neurogenic inflammation of pain-sensitive
'Department of Ophthalmology, Baskent University School of Medicine, Konya, Turkey

${ }^{2}$ Department of Ophthalmology, Baskent University School of Medicine, Ankara, Turkey

${ }^{3}$ Department of Neurology, Baskent University School of Medicine, Konya, Turkey

Correspondence:

A Karalezli,

BaskentUygulamave

ArastirmaHastanesi, GozHastaliklari, HocacihanMah, Saray Cad, No: 1, Konya 42080, Turkey

Tel: +90 3322570606 2208;

Fax: +90 3322570637.

E-mail: akaralezli@

yahoo.com

Received: 27 February 2014 Accepted in revised form: 10 August 2014 Published online: 26 September 2014 
cranial structures, hence worsening the pain. In migraine patients with aura, regional cerebral blood flow reduction starts during the aura and continues while the headache develops. At a certain point of the headache phase, regional cerebral blood flow increase to all level above normal: such an increase may well persist for a certain time after headache has disappeared. ${ }^{3}$ These unstable vascular abnormalities might also affect ocular blood flow, which can be assessed using color duplex imaging, laser speckle method, or Doppler flowmetry. ${ }^{4,5}$

However, because the choroid receives approximately 95\% of all ocular blood flow, changes in its structure could help to evaluate choroidal, thus ocular blood flow. ${ }^{6}$ In several recent studies, optical coherence tomography (OCT) was introduced as an effective tool for evaluating choroidal thickness. ${ }^{7,8}$ New-generation spectral domain OCT devices with enhanced scanning speed provide the opportunity to achieve high-resolution images and more accurate measurements. Thus, it has become possible to evaluate ocular tissues located at deeper levels than that of the retina, which was not possible with time domain OCT. $7,9,10$

In this study, we aimed to evaluate the choroidal thickness of migraine patients during acute migraine attacks and compare them with that of healthy controls.

\section{Materials and methods}

A total of 46 (24 female and 22 male) patients during acute migraine attacks referred from neurology clinic and 46 age- and gender-matched control (24 female and 22 male) subjects were consecutively enrolled in this study. The control cases had neither systemic nor ocular disease, nor any type of headache. To decrease the control selection bias, every age- and sex-matched control was consecutively selected after the inclusion of every eligible migraine patient in the study.

The study was approved by the Baskent University Institutional Review Board and Ethics Committee. The research adhered to the tenets of the Declaration of Helsinki, and a detailed written informed consent form was obtained before each individual's participation in the study. Patients were excluded if they had a history of ocular surface disorder, previous ocular surgery, or ocular injury; if they had a history of any systemic disease that could affect choroidal circulation (such as hypertension, diabetes, vasculitis, or renal failure); or if they were using any drug including analgesics, decongestants, or antihistamines. Moreover, in order to obtain clear images and to minimize the effect of axial length on choroidal thickness, patients with bestcorrected visual acuity worse than 20/20 and a refractive error higher than 1D were excluded from the study. ${ }^{11}$
All patients underwent a detailed ophthalmic examination, including visual acuity testing, refraction assessment, biomicroscopy, intraocular pressure measurement with non-contact tonometry, fundus examination, and choroidal thickness measurements by OCT. OCT scans were performed for each patient during the peak period of migraine attack. Headache pain intensity was measured on a four-point scale, where $0=$ no headache; $1=$ mild headache; $2=$ moderate headache; 3 = severe headache. This scale is recommended for use in migraine research by the International Headache Society. ${ }^{12}$ The patients were asked to grade their headaches according to this scale and called for OCT scans during a period of severe headache. All patients had bilateral headaches. To ensure consistency, only one eye of each patient (the right eye) was included. OCT scans were performed at the same time of day (09:00 hours) to minimize the effects of diurnal variation on choroidal thickness. ${ }^{13}$

All OCT scans were obtained with a high-speed, highresolution Fourier domain OCT device $(\lambda=840 \mathrm{~nm}$, $26000 \mathrm{~A}$-scans/s, and $5 \mu \mathrm{m}$ axial resolution) with Optovue RTVue software version 3.5 (Optovue Inc., Fremont, CA, USA). The choroidal thickness measurements were performed as follows: the scan pattern was the retina cross-line, which consists of two orthogonally oriented 6-mm lines containing 1024 A-scans. By automatically inverting the image, the chorioretinal interface became adjacent to the zero delay. The retina cross-line scan averages 32 frames, 16 per direction, without tracking. ${ }^{14}$ Each B-scan image is constructed from a number of repeated scans to reduce speckle noise and improve contrast; typical acquisition time of a constructed B-scan image is $1.25 \mathrm{~s}$. Choroidal thickness was measured using a built-in caliper tool adjusted perpendicularly from the outer edge of the retinal pigment epithelium to the choroid-sclera boundary at the fovea and at six other points, located at $1500 \mu \mathrm{m}$ temporal to the fovea, $1000 \mu \mathrm{m}$ temporal to the fovea, $500 \mu \mathrm{m}$ temporal to the fovea, $500 \mu \mathrm{m}$ nasal to the fovea, $1000 \mu \mathrm{m}$ nasal to the fovea and $1500 \mu \mathrm{m}$ nasal to the fovea (Figure 1).

Two masked technicians performed the choroidal thickness measurements. The average of the two measurements was used; the differences between readings of the masked physicians were found to be within $10 \mu \mathrm{m}$ of the mean. If the measurements did not agree within $10 \mu \mathrm{m}$, then they were repeated, and if the inconsistency persisted, a third masked reader (FCE) took a measurement. The interexaminer reproducibility of the choroidal thickness measurements was assessed by measuring the intraclass correlation coefficient (ICC).

Statistical analysis was performed with SPSS for Windows 11.6 (SPSS Inc., Chicago, IL, USA). Data were 


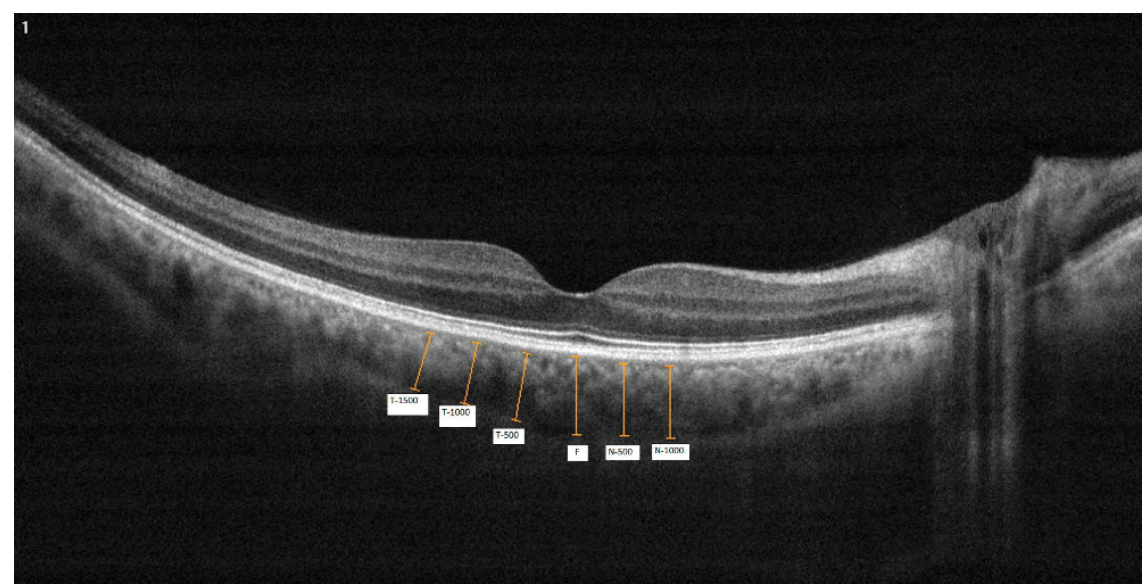

Figure 1 Example of choroidal thickness measurements. T-1500: Choroidal thickness at $1500 \mu \mathrm{m}$ temporal to the fovea; T-1000: Choroidal thickness at $1000 \mu \mathrm{m}$ temporal to the fovea; T-500: Choroidal thickness at $500 \mu \mathrm{m}$ temporal to the fovea; F: Choroidal thickness at fovea; N-500: Choroidal thickness at $500 \mu \mathrm{m}$ nasal to the fovea; N-1000: Choroidal thickness at $1000 \mu \mathrm{m}$ nasal to the fovea.

analyzed by independent sample T-test, and to quantify the reproducibility of manual re-measurements of the choroidal thickness in cases of alignment errors, the ICC was calculated. $P$ value $<0.05$ was considered statistically significant.

\section{Results}

The mean age of the migraine patients was $32 \pm 9.7$ years (range $=18-61$ years), and that of the controls was $33.2 \pm 7.1$ years (range $=18-61$ years). There was no statistically significant difference in age between the groups $(P=0.854)$.

The mean choroidal thickness was measured as $302.3 \pm 18.34 \mu \mathrm{m}$ in the control group and $356.3 \pm 21.46 \mu \mathrm{m}$ in the migraine patients during acute migraine attack. The mean choroidal thickness was statistically significant between groups $(P=0.000)$. Moreover, as shown in Table 1, the choroidal thicknesses of the patients during acute migraine attacks were all thicker than those of the controls in all eyes and at all seven measurement points.

The interexaminer ICC for the mean choroidal thickness was $0.938(95 \% \mathrm{CI}=0.908-0.985)$ and ICC was greater than 0.90 for all measurement points. Table 2 shows the comparison of the mean choroidal thickness measurements by two different observers in patients with acute migraine attack and control groups.

\section{Discussion}

The current study is a case-control study comparing subfoveal choroidal thickness between migraine patients during acute migraine attacks and healthy subjects. This study demonstrated the mean choroidal thickness was
Table 1 Mean choroidal thickness measurements in patients with acute migraine attack and control subjects

\begin{tabular}{lccc}
\hline & $\begin{array}{c}\text { Migraine patients } \\
\text { during acute migraine attack } \\
\text { Mean } \pm \text { SD }(\mu \mathrm{m})\end{array}$ & $\begin{array}{c}\text { Control subjects } \\
\text { Mean } \pm S D(\mu \mathrm{m})\end{array}$ & $\mathrm{P}$ value \\
\hline $\mathrm{N}-1500$ & $361.53 \pm 27.22$ & $305.23 \pm 28.24$ & 0.00 \\
N-1000 & $356.92 \pm 21.94$ & $302.23 \pm 19.95$ & 0.00 \\
N-500 & $358.38 \pm 18.94$ & $301.23 \pm 21.24$ & 0.00 \\
F & $362.76 \pm 21.23$ & $313.38 \pm 23.76$ & 0.00 \\
T-500 & $367.15 \pm 26.37$ & $318.01 \pm 25.88$ & 0.00 \\
T-1000 & $361.03 \pm 23.34$ & $305.42 \pm 20.81$ & 0.00 \\
T-1500 & $365.42 \pm 26.71$ & $310.03 \pm 24.52$ & 0.00 \\
\hline
\end{tabular}

$\mathrm{N}-1500$ : Choroidal thickness at $1500 \mu \mathrm{m}$ nasal to the fovea; N-1000: Choroidal thickness at $1000 \mu \mathrm{m}$ nasal to the fovea; N-500: Choroidal thickness at $500 \mu \mathrm{m}$ nasal to the fovea; F: Choroidal thickness at fovea; T-500: Choroidal thickness at $500 \mu \mathrm{m}$ temporal to the fovea; T-1000: Choroidal thickness at $1000 \mu \mathrm{m}$ temporal to the fovea; T-1500: Choroidal thickness at $1500 \mu \mathrm{m}$ temporal to the fovea.

significantly thicker in the eyes of patients during acute migraine attacks than that of the controls. These results suggest that choroidal thickness might be correlated with ocular blood flow, which in turn might be affected by rebound vasodilatation in patients with migraine in the acute attack period. ${ }^{1,15}$

Cranial blood vessels thus seem to be important players in the pathophysiology of migraine. In a recent study by Asghar et al, ${ }^{15}$ it was shown that migraine is associated with dilatation of extra and intracerebral arteries and the headache location is associated with the location of the vasodilatation. Furthermore, contraction of both extracerebral and intracerebral arteries is associated with amelioration of headache.

To date, no method has been defined as the 'gold standard' for evaluating choroidal blood flow. ${ }^{13}$ Since 
Table 2 Comparison of the mean choroidal thickness measurements of the migraine patients with acute migraine attack and control subjects between two different observers

\begin{tabular}{lcccc}
\hline & $\begin{array}{c}\text { Migraine patients during } \\
\text { acute migraine attack } \\
\text { Obsever 1 } \\
\text { Mean } \pm S D(\mu m)\end{array}$ & $\begin{array}{c}\text { Migraine patients during } \\
\text { acute migraine attack } \\
\text { Obsever 2 } \\
\text { Mean } \pm S D(\mu m)\end{array}$ & $\begin{array}{c}\text { Control subjects } \\
\text { Obsever 1 } \\
\text { Mean } \pm S D(\mu m)\end{array}$ & $\begin{array}{c}\text { Control subjects } \\
\text { Obsever 2 } \\
\text { Mean } \pm S D(\mu m)\end{array}$ \\
\hline N-1500 & $361.53 \pm 27.22$ & $360.47 \pm 24.41$ & $305.23 \pm 28.24$ & $304.23 \pm 21.23$ \\
N-1000 & $356.92 \pm 21.94$ & $357.34 \pm 23.42$ & $302.23 \pm 19.95$ & $304.27 \pm 23.15$ \\
N-500 & $358.38 \pm 18.94$ & $357.33 \pm 21.66$ & $301.23 \pm 21.24$ & $300.94 \pm 208.03$ \\
F & $362.76 \pm 21.23$ & $363.37 \pm 23.17$ & $313.38 \pm 23.76$ & $315.65 \pm 25.05$ \\
T-500 & $367.15 \pm 26.37$ & $366.11 \pm 22.55$ & $318.01 \pm 25.88$ & $317.13 \pm 23.78$ \\
T-1000 & $361.03 \pm 23.34$ & $363.12 \pm 28.39$ & $305.42 \pm 20.81$ & $306.77 \pm 19.94$ \\
T-1500 & $365.42 \pm 26.71$ & $363.51 \pm 22.43$ & $310.03 \pm 24.52$ & $311.13 \pm 22.13$ \\
\hline
\end{tabular}

$\mathrm{N}-1500$ : Choroidal thickness at $1500 \mu \mathrm{m}$ nasal to the fovea; N-1000: Choroidal thickness at $1000 \mu \mathrm{m}$ nasal to the fovea; $\mathrm{N}-500$ : Choroidal thickness at $500 \mu \mathrm{m}$ nasal to the fovea; F: Choroidal thickness at fovea; T-500: Choroidal thickness at $500 \mu \mathrm{m}$ temporal to the fovea; T-1000: Choroidal thickness at $1000 \mu \mathrm{m}$ temporal to the fovea; T-1500: Choroidal thickness at $1500 \mu \mathrm{m}$ temporal to the fovea.

their introduction in 2006, Fourier spectral domain OCT devices have provided high-speed scanning and highresolution images; therefore, an extremely vascular and dynamic tissue, such as the choroid, can be more accurately investigated. 7,16 As a highly vascular ocular structure, the choroid is directly influenced by intraocular and perfusion pressure; therefore its real-time high-definition images are more likely to demonstrate the real-time vascular status of this tissue in vivo. ${ }^{16}$

In previous studies, sildenafil citrate has been reported to increase choroidal thickness due to a vasodilatory effect of sildenafil citrate on the choroidal circulation. ${ }^{17}$ This correlation was further investigated by Kim et al ${ }^{18}$ using swept-scan high-frequency digital ultrasound to measure the ocular blood flow. In addition, in a latter study by Ulaş et al ${ }^{19}$ significant choroidal thinning was noted in chronic renal failure patients after hemodialysis. It is also reported in another recent study by Rishi et a ${ }^{20}$ that the choroidal thickness in eyes with polypoidal choroidal vasculopathy was found to be higher than the normal value, which leads to the argument that high mean ocular perfusion pressure could possibly have a role in the etiology of the disease. Moreover, in a recent study, cigarette smoking has been shown to be related with choroidal thickness decrease in otherwise healthy subjects. ${ }^{21}$

Komatsu et $a l^{22}$ evaluated 101 migraine patients during headache-free periods with the second derivative of the fingertip photoplethysmography (SDPTG) waveform and showed that migraine was complicated by peripheral circulation disorders of a vasoconstrictive nature in the attack-free period. In this study, we evaluated the choroidal thickness of migraine patients during the acute migraine attack period with OCT and found choroidal thickness is thicker than that of the controls, which in turn might be affected by rebound vasodilatation in migraine patients during an acute attack period.

There are several restrictions regarding our study, the main one being the low number of patients. A larger cohort would have allowed more meaningful analysis on the relation between the severity of acute migraine attacks and choroidal thickness; however, this can be achieved in a further study based on the preliminary findings of this study. Moreover, the design of the study with age- and sex-matched control group makes the results more valuable. The second limitation is the effect of diurnal rhythm of choroidal thickness (a change of approximately $20-30 \mu \mathrm{m}$ ), which was demonstrated in previous studies. ${ }^{13,22}$ To overcome this limitation and strengthen our study, the OCT examinations were performed at the same time of the day (0900 hours). The third limitation is that we analyzed only the patients with bilateral headaches. Also evaluation of patients with unilateral headaches and comparing the choroidal thicknesses of the eyes with each other in other studies with larger groups will give us a more definite conclusion. The last major limitation of this study is the fact that the choroidal thickness measurements had to be performed manually using the 'caliper function', which remains a potential cause for interobserver bias. To partly overcome this limitation, we have used seven different measurement points of each linear dimension and measured the thickness of the choroid via manual segmentation by two independent operators. In the future, reliable automated segmentation software for the sclera-choroidal interface may eliminate significant differences between graders.

Despite these limitations, to the best of our knowledge, our study is the first to investigate choroidal thickness in migraine patients during an acute attack period. Further studies with larger groups (with unilateral and bilateral 
headaches) and longer follow-up periods are necessary to come to a more definite conclusion.

\section{Summary}

\section{What was known before}

- The purpose of this study was to assess choroidal thickness in migraine patients during acute migraine attacks and compare them with healthy controls, using spectral domain optical coherence tomography (OCT).

\section{What this study adds}

- The increased choroidal thickness of the migraine patients during acute migraine attacks might be related to the vascular pathology of the disease.

\section{Conflict of interest}

The authors declare no conflict of interest.

\section{References}

1 Panconesi A, Bartolozzi ML, Guidi L. Migraine pain: reflections against vasodilatation. J Headache Pain 2009; 10: 317-325.

2 Charles A. Vasodilatation out of the picture as a cause of migraine headache. Lancet Neurol 2013; 12: 419-420.

3 Lauritzen M. Cerebral blood flow in migraine and cortical spreading depression. Acta Neurol Scand Suppl 1987; 113: $1-40$.

4 Sugiyama T, Araie M, Riva CE, Schmetterer L, Orgul S. Use of laser speckle flowgraphy in ocular blood flow research. Acta Ophthalmol 2010; 88: 723-729.

5 Portman N, Gugleta K, Kochkorov A, Polunina A, Flammer J, Orgul S. Choroidal blood flow response to isometric exercise in glaucoma patients and patients with ocular hypertension. Invest Ophthalmol Vis Sci 2011; 52: 7068-7073.

6 Alm A. Ocular circulation. In: Hart WM Jr (ed). Adler's Physiology of the Eye, 9th edn. Mosby: St Louis, MO, USA, 1992, pp 198-227.

7 Manjunath V, Taha M, Fujimoto JG, Duker JS. Choroidal thickness in normal eyes measured using Cirrus-HD optical coherence tomography. Am J Ophthalmol 2010; 150: 325-329.

8 Maul EA, Friedman DS, Chang DS et al. Choroidal thickness measured by spectral domain optical coherence tomography: factors affecting thickness in glaucoma patients. Ophthalmology 2011; 118: 1571-1579.

9 Drexler W, Fujimoto JG. State-of-the-art retinal optical coherence tomography. Prog Retin Eye Res 2008; 27: 45-88.

10 Sander B, Larsen M, Thrane L, Hougaard JL, Jongersen TM. Enhanced optical coherence tomography imaging by multiple scan averaging. Br J Ophthalmol 2005; 89: 207-212.

11 Fujiwara T, Imamura Y, Margolis R, Slakter JS, Spaide RF. Enhanced depth imaging optical coherence tomography of the choroid in highly myopic eyes. Am J Ophthalmol 2009; 148: 445-450.

12 Tfelt-Hansen P, Pascual J, Ramadan $\mathrm{N}$ et al. International headache society clinical trials subcommittee. Guidelines for controlled trials of drugs in migraine: third edition. A guide for investigators. Cephalalgia 2012; 32: 6-38.

13 Tan CS, Ouyang Y, Ruiz H, Sadda SR. Diurnal variation of choroidal thickness in normal, healthy subjects measured by spectral domain optical coherence tomography. Invest Ophthalmol Vis Sci 2012; 53: 261-266.

14 Polska E, Polak K, Luksch A et al. Twelve hour reproducibility of choroidal blood flow parameters in healthy subjects. Br J Ophthalmol 2004; 88: 533-537.

15 Asghar MS, Hansen AE, Amin FM et al. Evidence for a vascular factor in migraine. Ann Neurol 2011; 69: 635-645.

16 Manjunath V, Goren J, Fujimoto JG, Duker JS. Analysis of choroidal thickness in age-related macular degeneration using spectral-domain optical coherence tomography. Am J Ophthalmol 2011; 152: 663-668.

17 Vance S, Imamura Y, Freund KB. The effects of sildenafil citrate on choroidal thickness as determined by enhanced depth imaging optical coherence tomography. Retina 2011 31: 332-335.

18 Kim DY, Silverman RH, Chan RVP et al. Measurement of choroidal perfusion and thickness following systemic sildenafil (Viagra). Acta Ophthalmol 2013; 91: 183-188.

19 Ulaş F, Doğan U, Keles A et al. Evaluation of choroidal and retinal thickness measurements using optical coherence tomography in non-diabetic haemodialysis patients. Int Ophthalmol 2013; 33: 533-539.

20 Rishi P, Rishi E, Mathur G et al. Ocular perfusion pressure and choroidal thickness in eyes with polypoidal choroidal vasculopathy, wet-age-related macular degeneration, and normals. Eye 2013; 27: 1038-1043.

21 Sizmaz S, Küçükerdönmez C, Pinarci EY. The effect of smoking on choroidal thickness measured by optical coherence tomography. Br J Ophthalmol 2013; 97: 601-604.

22 Komatsu K, Fukutake T, Hattori T. Fingertip photoplethysmography and migraine. J Neurol Sci 2003; 216: 17-21. 